\title{
Prevalence Study (2017-2020) of Heart Failure among Dogs of Hyderabad, Telangana State
}

\author{
E. Aravind Rao', V.V.V. Amruth Kumar², P. Nagaraj ${ }^{3}$, K. Satish Kumar
}

10.18805/IJAR.B-4789

\begin{abstract}
Background: Heart failure that is caused by various cardiac diseases like valvular disease, dilated cardiomyopathy and pericardial diseases are progressive and chronic in nature, but occur as acute form wherein the clinician has to respond quickly both in its diagnosis and treatment.

Methods: The present study that was carried out to assess the prevalence of heart failure has included 309 dogs of various breed, gender and age. Clinical evaluation was followed by electrocardiography using smart phone-based ECG, thoracic radiography and $2 \mathrm{~d}$-echocardiography to diagnose the various causes of heart failure.

Result: All of these cases were exhibiting similar manifestations like generalized weakness, poor physical activity, respiratory distress, dyspnoea at rest, cough and lack of sleep, that were suggestive of heart failure. ECG, thoracic radiography and echocardiographic evaluation revealed 146/309 cases as suffering with various cardiac diseases viz., valvular diseases, dilated cardiomyopathy and pericardial diseases that resulted in heart failure. However, the remaining 163/309 dogs were diagnosed with respiratory disorders (69), renal insufficiency (57), hepatic insufficiency (26), pyometra (07) and anemia (04). The overall prevalence of heart failure was observed to be $1.55 \%$ (146) among the total dogs (9369) presented with systemic diseases, while it was $47.25 \%$ (146) among dogs (309) which were exhibiting clinical manifestations suggestive of heart failure. Similarly, the heart failure was recorded as highest prevalent among Labrador and lowest among Daschund. Dogs aged between 10-14 years are more vulnerable for these cardiac diseases when compared to various other age group individuals and males are more at risk.
\end{abstract}

Key words: Age, Breed, Dog, Failure, Gender, Heart, Prevalence.

\section{INTRODUCTION}

Heart disease in dogs is almost as common as it is in humans which needs an immediate attention in diagnosis and management, as the delay could result in a grave prognosis or life-threatening condition. Many times the condition is detected too late until pronounced signs appear (Martin et al. 2009). Though research and documentation on various cardiac diseases in canines are extensively available all over the world, information in India is scarce. Lack of awareness and knowledge by the owner and inadequate diagnostic facility could be probable reasons (Devi et al. 2009). However, in due course, provoked numerous investigations leading to advancements such as, access to echocardiographic facility in veterinary practice which has revolutionized the field of cardiology by aiding the diagnosis and management of cardiac diseases in dogs. Even though, cardiac disorders are progressive and chronic in nature, they occur as acute form wherein the clinician has to respond quickly both in its diagnosis and treatment (Kumar et al. 2021). It is estimated that approximately $10 \%$ of dogs presented to Primary Veterinary care have heart disease. Chronic Valvular Heart Disease is the most common heart disease observed in dogs in many parts of the world, that accounts for approximately $75 \%$ of heart disease cases seen in veterinary practice (Atkins et al. 2009).

\section{MATERIALS AND METHODS}

All the adult dogs of various breed, age and gender that were presented to the non-infectious medicine ward of Teaching
Department of Veterinary Medicine, College of Veterinary Science, PV Narsimha Rao Telangana Veterinary University, Rajendranagar, Hyderabad-500 030, Telangana, India.

${ }^{1}$ Modern Abattoir Facility, Chengicherla-500 039, Telangana, India. ${ }^{2}$ Department of Veterinary Medicine, College of Veterinary Science, Korulta-505 326, Telangana, India.

${ }^{3}$ Veterinary Hospital, Bhoiguda-500 003, Telangana, India.

Corresponding Author: K. Satish Kumar, Department of Veterinary Medicine, College of Veterinary Science, PV Narsimha Rao Telangana Veterinary University, Rajendranagar, Hyderabad-500 030, Telangana, India. Email:drsatish.ksk@gmail.com

How to cite this article: Rao, E.A., Amruth Kumar, V.V.V., Nagaraj, P. and Kumar, K.S. (2022). Prevalence Study (2017-2020) of Heart Failure among Dogs of Hyderabad, Telangana State. Indian Journal of Animal Research. DOI: 10.18805/IJAR.B-4789.

Submitted: 24-09-2021 Accepted: 18-12-2021 Online: 29-01-2022

Veterinary Clinical Complex, College of Veterinary Science, Rajendranagar and Veterinary Hospital Bhoiguda, Secunderabad during 2017 -2020, were included for the present study. Further, those dogs that had a history and manifestations suggestive of heart failure were considered for detailed evaluation. All these dogs were clinically and physically evaluated in detail and later subjected for specific tests like electrocardiography, thoracic radiography and echocardiography for confirmation of cardiac disease, to access its prevalence. 
Electrocardiographic evaluation of heart failure suspected dogs was carried out using smart phone based ECG (sECG), that can either be fixed onto the back of an iPhone or any android phone or can be used separately by placing the phone close to the electrodes. The left cardiac area (between 3-5 ICS) is trimmed, moistened with conduction gel or alcohol and the smart phone device was positioned on the left chest wall, just caudal to the olecranon (precordial area), with a slightly dorso-cranial / ventro-caudal orientation (Kumar et al. 2021). A single lead (L II) sECG was recorded for 30 seconds in an unsedated dog either on its right lateral recumbency or in standing position, at 25 $\mathrm{mm} / \mathrm{s}$. The recorded $\mathrm{sECGs}$ were automatically digitised by the device and stored as a PDF, which can subsequently be analysed on a computer and interpreted as described by Tilley (1992). Thoracic radiographs were taken on left lateral and ventro-dorsal views at the peak inspiration. Transthoracic echocardiograms were obtained with the unsedated dogs in right lateral recumbency (Allworth et al. 1995). Transducer is located parasternally between right third and sixth intercostal spaces between sternum and costochondral junction (Thomas et al. 1994). M-mode recordings were taken at the high papillary level to measure various left ventricle dimensions (AllWorth et al. 1995) and pulsed wave Doppler and colour flow Doppler was also done to confirm valvular disease (Dominique and Marc-Andre, 2008).

\section{RESULTS AND DISCUSSION}

During the study period (2017 - 2020), a total of 9369 adult dogs with the history and signs suggestive of various systemic diseases were presented and out of which, 309 were exhibiting the manifestations indicative of heart failure. All these cases when subjected for detailed analysis using ECG, thoracic radiography and echocardiography, 146/309 cases were diagnosed for heart failure. Whereas, the remaining 163/309 dogs were diagnosed with respiratory disorders (69), renal insufficiency (57), hepatic insufficiency (26), pyometra (07) and anemia (04). The overall prevalence of heart failure was observed to be $1.55 \%$ (146) among the dogs presented with systemic diseases (9369). It was $47.25 \%$ (146) among dogs which were exhibiting clinical manifestations (309) suggestive of heart failure (Table 1 and Fig1). All these dogs that were diagnosed for heart failure were showing similar manifestations viz., exercise intolerance, dyspnoea at rest, nocturnal cough, insomnolence, anorexia etc. are associated with left main stem bronchus compression and pulmonary edema (Kumar and Kumar, 2021; Turgut et al. 2017). Coughing, which is predominant in these cases might be a result of pressure on the left mainstem bronchus caused by left atrial enlargement (Satish et al. 2011), rather than a result of pulmonary edema (Carr, 2004). Lethargy and reduced physical activity could be due to diminished oxygen delivery to the tissues associated with reduced cardiac output (Freeman, 2012). Hypostastic congestion of lungs and pulmonary edema lead to dyspnoea at rest (Ristic, 2004). Other pathophysiological events that help for the development of the clinical manifestation of the disease may include an increase in end-diastolic volume that subsequently increase myocardial stress, thinning of the myocardium and rounded left ventricle apex (Domanjko et al. 2002).

Abnormalities associated with P, QRS and T that were detected among these heart failure dogs include, wide and / or elevated $\mathrm{P}, \mathrm{R}$ and $\mathrm{QRS}$ waves, atrial fibrillation and ventricular premature complexes. Auscultation of cardiac area revealed murmur of varied intensity (G I to IV). The systolic murmur on auscultation is a hallmark of the valvular disease, where the intensity varies with severity of disease and can be a significant among aged dogs (Kumar and Kumar, 2021). Presence of S3 sound with an increased intensity of S1 and decreased S2 sound on left cardiac area are significant physical findings of MMVD (Hägström et al. 1995). Bulge of left atrium, elevation of trachea, pulmonary congestion and vertebral heart score ranging from 11.8 to 13.2, were the significant radiographic abnormalities of the present heart disease cases. Radiographic assessment of cardiac size is another important guide to diagnose cardiac disease which also be used to monitor the severity of disease (Thrall, 2007), though it is difficult to make accurate subjective assessment whether the cardiac silhouette is enlarged or misshapen (Nakayama et al. 2001). However, thoracic radiography is considered the clinical "gold standard" method for the diagnosis of heart failure (Balbarini et al. 1991). Radiographically estimated vertebral heart score of more than 12 dogs along with plasma NT pro-BNP $(>1500 \mathrm{pmol} / \mathrm{L})$ are the significant aspects of threatened

Table 1: Prevalence of various systemic diseases in dogs $(n=309)$.

\begin{tabular}{lcc}
\hline Systemic disorder & No. of dogs & Percentage \\
\hline Heart failure & 146 & 47.25 \\
Respiratory disorders & 69 & 22.33 \\
Renal insufficiency & 57 & 18.47 \\
Hepatic insufficiency & 26 & 08.41 \\
Pyometra & 07 & 03.24 \\
Anemia & 04 & 01.29 \\
Total & 309 & 100 \\
\hline
\end{tabular}

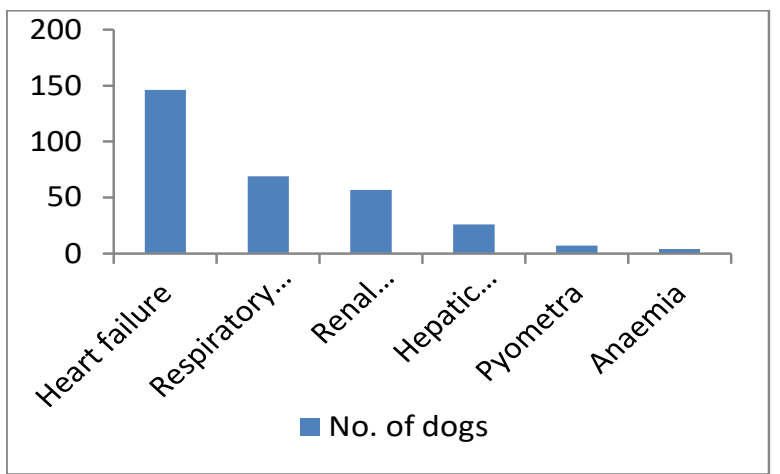

Fig 1: Prevalence of systemic diseases in dogs. 
heart failure among chronic mitral valve diseased dogs (Bonagura and Schober, 2009). The common echocardiographic abnormalities noticed among these 146 heart failure dogs include hypokinesis of interventricular septum, dilated atria, ventricles, degeneration of mitral valve leaflets, kinking, prolapse and flail nature of mitral valve leaflets and pericardial effusion. Based on these findings dilated cardiomyopathy, valvular disease and pericardial effusion were diagnosed as the causes of heart failure. Though, clinical and physical examination, thoracic radiography, electrocardiography, echocardiography and cardiac biomarkers are available diagnostic tools (Nakayama et al. 2001), non invasive techniques like radiography and echocardiography are the most common diagnostic procedures used in confirming the heart disease in dogs (Kumar et al. 2021). The degree of prevalence of heart failure in dogs noted in the current study is in agreement with that of Satish et al., (2011) who reported an overall occurrence of cardiac disease in dogs as $1.65 \%$ and it was $48.6 \%$ among dogs exhibiting clinical manifestations suggestive of heart disease. Haritha et al., (2017) reported overall prevalence of cardiac disease in dogs as $1.77 \%$ and the prevalence among the dogs exhibiting clinical signs suggestive of heart disease was $56.21 \%$. The prevalence of DCM in dogs was reported to be between $0.45 \%$ and $1.1 \%$ by the COVE study group (1995). However, higher prevalence rate was also reported by other workers as $11.3 \%$ (Detweiler and Patterson, 1965), 10\% (Atkins et al. 2009) and 5\% (Ettinger and Suture, 1970). Baumgartner and Glaus, (2004) reported prevalence rates of $49.40 \%$ (valvular disease), $21.10 \%$ (DCM), $12.4 \%$ (pericardial effusion) and $0.60 \%$ (hypertrophic cardiomyopathy). Low prevalence of $0.68 \%$ was reported by Kumar et al., (2021) and $0.5 \%$ by Sisson et al. (2000). Deepti et al., (2016) reported the occurrence of CHF in dogs as $0.45 \%$, MVD as $0.11 \%$ and DCM as $0.34 \%$, respectively. The difference in the prevalence rates of the heart disease in dogs could be due to the diverse populations being screened in each study with respect to age, breed, gender and geographical location.

The various cardiac diseases viz., dilated cardiomyopathy, valvular diseases and pericardial diseases that are responsible for heart failure in the present study are more predominantly recorded $(51.37 \%)$ in dogs between 10 to 14 year. This was followed by $32.87 \%$ among 5 to 10 year old dogs and $10.27 \%$ among those aged more than 14 years and less prevalence rate of $05.48 \%$ was recorded in dogs of less than 5 years (Fig 2). These findings are near agreement to the findings of Satish et al., (2011) who reported that age wise occurrence of cardiac disease in dogs was $61.24 \%$ in 10 to 14 years of age, $21.12 \%$ in $5-9$ years, $12.18 \%$ in more than 14 years and $5.46 \%$ in less than 5 years of age. Haritha et al., (2017) reported the highest prevalence of DCM in dogs between 05 to 10 years of age (50.44\%), whereas Deepti et al. (2016) reported the age wise highest prevalence in $5-10$ years of age (54\%), Jeyaraja et al., (2008) reported the average age as $8.5 \pm 0.5 \mathrm{yrs}$ and whereas, Tidholm et al., (2001) stated that age at onset of clinical signs varies considerably, although most dogs are initially presented at the age of five to seven years. The current findings are in agreement with Kumar et al., (2021) who reported higher prevalence of cardiovascular diseases in aged dogs, stating that the ageing process affects the heart and the blood vessels by depleting cardiovascular reserve and altering the responses to various diseases and medications. Ageing has selective effects on peripheral vascular function, including a reduced arterial dilating response to catecholamines.

With respect to breed wise prevalence study, the highest number of various cardiac diseases were detected among Labrador retriever (39.72\%) followed by Pomeranian (26.71\%), Spitz (13.01\%), Doberman (06.16\%), Boxer (04.11\%), Cross breed (04.11\%), Cocker spaniel $(02.74 \%)$, Pug $(2.05 \%)$ and lowest number of cases $(01.37 \%)$ that were diagnosed for for heart failure was recorded in Dachshund (Fig 3). This pattern of variation in prevalence could be attributed to the predominance of a larger number of dogs of Labrador retriever and Pomeranian breeds in the geographic place where the present study was carried out. It is also evident that higher prevalence of heart failure in certain breeds that could be possibly related to inherited transmission of heart disease in these breeds. Dukes (2000)

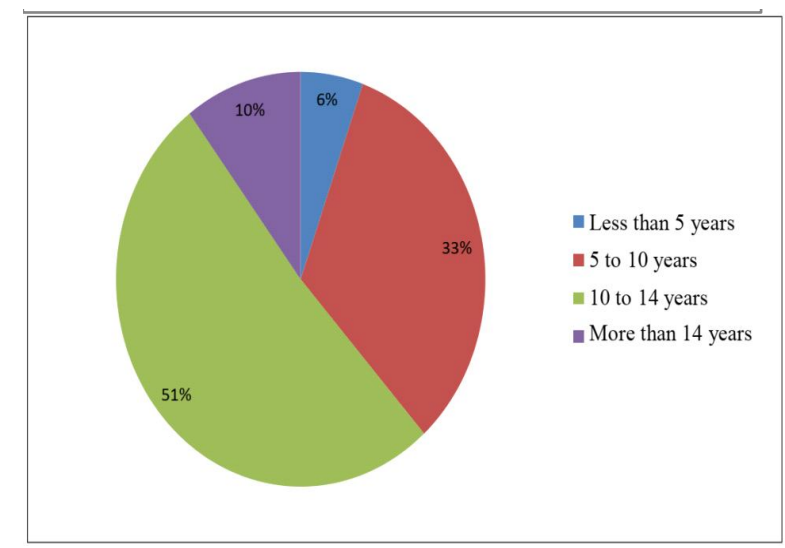

Fig 2: Age-wise prevalence of heart failure.

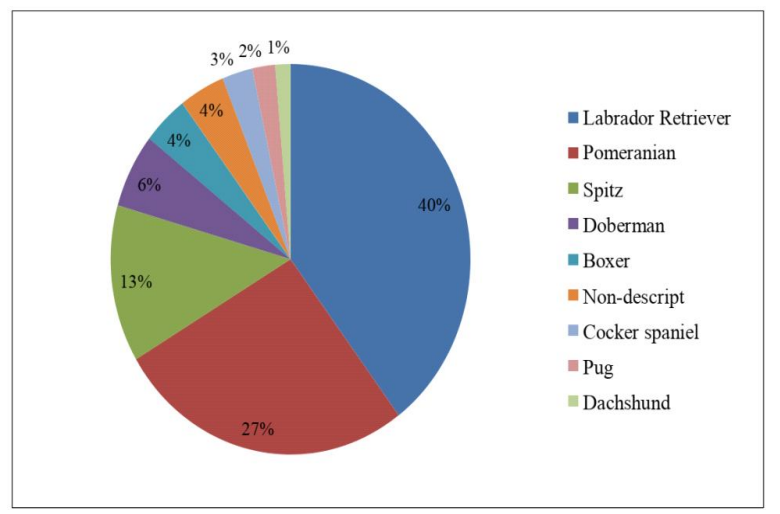

Fig 3: Breed-wise prevalence of heart failure in dogs. 


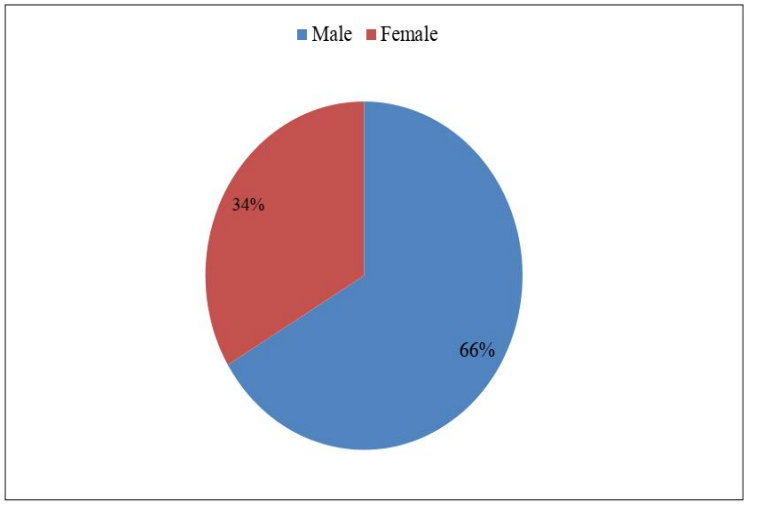

Fig 4: Gender-wise prevalence of heart failure in dogs.

detected the familial predisposition to DCM in certain breeds like Doberman pinscher and Boxers. Dilated Cardiomyopathy occurs with significantly greater frequency in pure bred dogs than cross breed dogs (Mc Ewan et al. 2003). Clinical forms of DCM have been described in giant breed dogs (Tidholm, 2001), Doberman pinscher (Domanjko et al. 2002) and Boxers (Valentina et al. 2011). Further, canine DCM has been documented in many breeds, although uncommon in dogs weighing less than $15 \mathrm{~kg}$ (Fox, 1998).

The prevalence of heart failure was relatively higher in male dogs $(66.44 \%)$ when compared to that of females $(33.56 \%)$ and these findings are in accordance with that of Deepti et al., (2016), Haritha et al., (2017) and Satish et al., (2011), who documented greater prevalence of cardiac disease in males than in females. Sisson et al., (2000) also observed that the males were nearly twice as often affected than females with respect to the development of heart failure or sudden death with dilated cardiomyopathy and Martin et al., (2009) reported males are nearly four times more affected than female dogs (Fig 4).

\section{CONCLUSION}

Dogs presented with the history and signs like dyspnoea at rest, exercise intolerance and low physical activity should be considered for cardiac evaluation by ECG, thoracic radiography and echocardiography. Even though, the heart failure is common among all the breed and gender of adult dogs, more emphasis in evaluation should be given among Labrador retriever and also among those dogs that are more than 10 years.

\section{REFERENCES}

Allworth, S.M., Church, D.B., Maddison, J.E., Einstein, R., Brennar, P., Anssein, N.A. and Mathews, R. (1995). Effect of enalapril in dogs with pacing induced heart failure. American Journal of Veterinary Research. 56: 86-94.

Atkins, C., Bonagura, J., Ettinger, S., Fox, P., Gordon, S., Haggstrom, J., Hamlin, R., Keene, B., Luis Fuentes, V.and Stepien, R. (2009). Guidelines for the diagnosis and treatment of canine chronic valvular heart disease. Journal of Veterinary Internal Medicine. 23: 1142-1150.
Balbarini, A., Limbruno, U., Bertoli, D., Tartarini, G. and Baglini, R. (1991). Evaluation of pulmonary vascular pressures in cardiacpatients: The role of the chestroentgenogram. Journal of Thoracic Imaging. 6: 62-68

Baumgartner, C. and Glaus, T.M. (2004). Acquired cardiac diseases in the dog: a retrospective analysis. Schweizer Archivfur Tierheilkunde. 146(9): 423-430

Bonagura, J.D. and Schober, K.E. (2009). Can ventricular function be assessed by echocardiography in chroniccanine mitral valve disease? Journal of Small Animal Practice. 50(1): 12-24.

Carr, A.P. (2004). Cardiac Disease in Geriatric Dogs and Cats. In: Geriatrics and Gerontology of the Dog and Cat. WB Saunders, pp. 127-148.

Deepti, B.R., Yathiraj, S., Ramesh, P.T., Ranganath, L. and Narayanaswamy, H.D. (2016). Diagnosis and treatment of Congestive Heart Failure in Canines. Intas Polivet.17: 129-131.

Detweiler, D.K. and Patterson, D.F. (1965). The Prevalence and Types of Cardiovascular Disease in Dogs. Annals of New York Academy of Sciences. 147: 318.

Devi, S., Jani, R.G., Anne, F.K. and Singh, R.D. (2009). Study on clinical symptoms in canine cardiac diseases. Vet World. 2(8): 307-309.

Domanjko, P.A., Stabej, P. and Zemva, A. (2002). Dilated cardiomyopathy in Doberman Pinschers: Survival, Causes of Death and a Pedigree Review in a Related Line. Journal of Veterinary Cardiology. 4(1): pp 17-24.

Dominique, P. and Marc-Andre d' Anjou. (2008). Atlas of small Animal Ultrasonography, I edn., Blackwell Publishing, Australia.

Dove, R.S. (2001). Nutritional therapy in the treatment of heart disease in dogs. Alternatives of Medical Review. 6: 3845.

Dukes McEwan, J. (2000). Canine Dilated Cardiomyopathy 2. Pathophysiologyand treatment. In Practice. 22: pp 620626.

Ettinger, S.J. and Suter, P.F. (1970). Canine Cardiology, W.B. Saunders Co., Philadelphia.

Fox, P.H. (1998). Canine Myocardial Disease. In: Canine and feline cardiology. Churchill Livingstone, NewYork, pp 467-487.

Freeman, L.M. (2012). Cachexia and sarcopenia: Emerging syndromes of importance in dogs and cats. Journal of Veterinary Internal Medicine. 26: 3-17.

Hägström, J., Kvart, C. and Hansson, K. (1995). Heartsounds and Murmurs: Changes related to severity ofchronic valvular disease in the Cavalier King Charles Spaniel. Journal of Veterinary Internal Medicine. 9: 75-85.

Haritha, G.S., Kumar, K.S., Ayodhya, S., Kumar, V.V.V.A. (2017). Prevalence of Cardiac Disorders in Canines- A Clinical Study. Intas Polivet. 18(1): 148-153.

Jeyaraja, K., Gowri, B., Nambi, A.P. and Vasu, K. (2008). Echocardiographic evaluation of dilated cardiomyopathy in dogs. Indian Veterinary Journal 85: 983-984.

Kumar, S.K., Kumar, A.V.V.V. and Aravind, E. (2021). Smart phone based electrocardiography in dogs-A newer concept in veterinary cardiology. Journal of Animal Research. 11(3): 393-400. 
Kumar, K.S. and Kumar, V.V.V.A. (2021). Diagnosis of Myxomatous Mitral Valve Disease (MMVD) in Aged Dogs. Indian Journal of Animal Research. DOI: 10.18805/IJAR.B4403.

Martin, M.W.S., Johnson, M.J.S. and Celona, B. (2009). Canine dilated cardiomyopathy: A retrospective study of signalment, presentation and clinical fundings in 369 cases. Journal of Small Animal Practice. 50: 23-29.

McEwan, J.D., Michele, B., Anna, T. andrea, C.V. and Haggstrom, J. (2003). Proposed Guidelines for the Diagnosis of canine idiopathic dilated cardiomyopathy. The ESVC Taskforce for canine dilated cardiomyopathy. Journal of Veterinary Cardiology. 5(2): 7-19.

Nakayama, H., Nakayama, T. and Hamlinxya, R.L. (2001). Correlation of cardiac enlargement as assessed by vertebral heart size and echocardiographic and electrocardiographic findings in dogs with evolving cardiomegaly due to rapid ventricular pacing. Journal of Veterinary Internal Medicine. 15(3): 217-221.

Ristic, J. (2004). Clinical assessment of the dog with suspected cardiac disease. In Practice. 26: 192-199.

Satish, K.K. Tirumala, R.D.S. and Nagaraj, P. (2011). Dilated Cardiomyopathy in dogs: A study for 3 years (2006 to 2009). Vet scan. 6;2: Article 93.
Sisson, D.D., Thomas, W.P. and Keene, B.W. (2000). Primary myocardial disease in the dog. In: Text book of Veterinary Internal Medicine, [Ettinger S.E. and Feldman F.C. (eds)]: $5^{\text {th }}$ edn, W.B. Saunders Co., Philadelphia, pp 874-896.

Thomas, P.W., Gaber, C.E., Jacobs, G.J., Kaplan, P.M., Lombard, C.W., Moise, N.S. and Moses, B.L. (1994). Recommendations for standards in transthoracic two-dimensional echocardiography in the dog and cat. Veterinary Radiology and Ultrasound. 35: 173-178.

Thrall, D.E. (2007). Veterinary Radiology. $5^{\text {th }}$ edn., Philadelphia, Saunders Elsevier, pp.479-485.

Tidholm, A., Haggstrom, J., Borgarelli, M. and Tarducci, A. (2001). Canine Idiopathic Dilated Cardiomyopathy. Part I: Aetiology, Clinical Characteristics, Epidemiology and Pathology. The Veterinary Journal. 162: 92-107.

Tilley, L.P. (1992). In: Essentials of canine and feline electrocardiogrphy, Tilley, L.P., (ed): $3^{\text {rd }}$ edn, Lea and Fabiger, Philadelphia. 23-156.

Turgut, K. (2017). Klinik Kedi ve Köpek Kardiyolojisi. Nobel Tıp Kitabevleri Tic. Ltd. Şti., İstanbul.

Valentina, P., Michael, J., Stafford J., Elisabetta, S., Paola, G.B. and Martin, W.S. (2011). Cardiomyopathy in Boxer dogs: A retrospective study of the clinical presentation, diagnostic findings and survival. Journal of Veterinary Cardiology. 13: 45-55. 